\title{
Severity assessment for lower respiratory tract infections: potential use and validity of the CRB-65 in primary care
}

\author{
*Nick A Francisa, Jochen W Cals ${ }^{b}$, Christopher C Butlera, Kerenza Hood ${ }^{a}$, Theo Verheijc, \\ Paul Little ${ }^{d}$, Herman Goossens ${ }^{\mathrm{e}}$, Samuel Coenen ${ }^{\mathrm{e}, \mathrm{f}}$ on behalf of the GRACE Project Group
}

\footnotetext{
a Department of Primary Care and Public Health, School of Medicine, Cardiff University, Cardiff, UK

${ }^{\mathrm{b}}$ Department of General Practice, CAPHRI School for Public Health and Primary Care, Maastricht University Medical Center, Maastricht, The Netherlands

c Julius Center for Health Sciences and Primary Care, University Medical Center Utrecht, Utrecht, The Netherlands

' Primary Care Medical Group, Community Clinical Sciences Division, University of Southampton School of Medicine, Southampton, UK

e Laboratory of Medical Microbiology, Vaccine and Infectious Disease Institute (VAXINFECTIO), University of Antwerp-Campus Drie Eiken, Antwerp, Belgium

Centre for General Practice, Vaccine and Infectious Disease Institute (VAXINFECTIO), University of Antwerp-Campus Drie Eiken, Antwerp, Belgium
}

Received 13th May 2011; revised 11th July 2011; accepted 13th July 2011; online 21st September 2011

\begin{abstract}
Aims: To explore the potential use of the CRB-65 rule (based on Confusion, Respiratory rate, Blood pressure and age $>65$ years) in adults with lower respiratory tract infection (LRTI) in primary care.

Methods: Primary care clinicians in 13 European countries recorded antibiotic treatment and clinical features for adults with LRTI. Patients recorded daily symptoms. Multilevel regression models determined the association between an elevated CRB-65 score and prolonged moderately severe symptoms, hospitalisation, and time to recovery. Sensitivity analyses used zero imputation.

Results: Respiratory rate and blood pressure were recorded in $22.7 \%$ and $31.9 \%$ of patients, respectively. A total of 2,690 patients completed symptom diaries. The CRB-65 could be calculated for 339 (12.6\%). A score of $\geq 1$ was not significantly associated with prolonged moderately severe symptoms (odds ratio (OR) $0.42,95 \% \mathrm{Cl} 0.04$ to 4.19 ) or hospitalisations $(\mathrm{OR} 3.12,95 \% \mathrm{Cl} 0.16$ to 60.24$)$, but was associated with prolonged time to self-reported recovery when using zero imputation (hazard ratio ( $\mathrm{HR}) 0.75,95 \% \mathrm{Cl} 0.64$ to 0.88).

Conclusions: Respiratory rate and blood pressure are infrequently measured in adults with LRTI. We found no evidence to support using the CRB-65 rule in the assessment of LRTI in primary care. However, it is unclear whether it is of value if used only in patients where the primary care clinician suspects pneumonia.

(C) 2012 Primary Care Respiratory Society UK. All rights reserved.

NA Francis et al. Prim Care Respir J 2012; 21(1): 65-70

http://dx.doi.org/10.4104/pcrj.2011.00083
\end{abstract}

Keywords risk assessment, lower respiratory tract infections, primary health care, cough, prognosis, CRB-65

\section{See linked editorial by Marsden \& Woodhead on pg 11}

\section{Introduction}

Community-acquired lower respiratory tract infection (LRTI) is one of the most frequent diagnoses in medical care. Most cases are managed in primary care where an extensive diagnostic work-up for all patients is neither feasible nor cost-effective.
Identifying which of the many presenting patients are likely to experience an unusual or prolonged illness course is helpful in setting realistic expectations about recovery and advising on appropriate help-seeking. Community-based clinicians rely on clinical judgement to determine which patients are likely to experience a more complicated or prolonged course. However, there is insufficient evidence on the diagnostic value of signs,

\footnotetext{
* Corresponding author: Dr Nick A Francis, Department of Primary Care and Public Health, School of Medicine, Cardiff University, Heath Park, Cardiff CF14 4XN, UK. Tel: +44 (0)29 20687133 Fax: +44 (0)29 20687611 E-mail: francisna@cf.ac.uk
} 
symptoms and rapid or near patient tests to identify reliably patients likely to have a worse prognosis. ${ }^{1}$ Clinician uncertainty and patient expectations for antibiotic treatment contribute to substantial overuse of antibiotics in most European countries. This results in substantial direct and indirect costs and serious unintended consequences including side effects from unnecessary antibiotics ${ }^{2}$ and, most notably, a rise in bacterial resistance. ${ }^{3,4}$

To counteract unnecessary antibiotic use and associated pressure for selecting bacterial resistance, primary care clinicians need valid feasible tools to improve the quality of their management decisions. Prognostic models that are valid in primary care can help clinicians tailor their management strategies to the risk of complications or prolonged illness. Indeed, the development and evaluation of strategies or tools to help differentiate between serious and self-limiting LRTIs was described as a key research need by the International Primary Care Respiratory Group. ${ }^{5}$

The CRB-65 rule, based on Confusion, Respiratory rate, Blood pressure and age $\geq 65$, was developed to predict the risk of admission to the intensive care unit and mortality in hospitalised patients with community-acquired pneumonia (CAP). ${ }^{6.7}$ Although developed in the hospital setting, it has also been promoted as a tool for guiding decisions about hospital admission in patients with CAP initially managed in the community. ${ }^{8-11}$ However, its validity in this setting has not been adequately assessed. There have been three recent systematic reviews of evidence for using severity assessment rules in the management of CAP..$^{10-12}$ Two of these excluded patients who had not had a chest $x$-ray. ${ }^{10,11}$ This limited the applicability of the findings as an $x$-ray is not performed for most patients with LRTI managed in the community. The third review compared the use of the CRB-65 to predict 30-day mortality in hospital and community settings, and found that the rule over-predicted the probability of mortality in community settings. ${ }^{12}$ However, while studies have evaluated the use of CRB-65 in outpatient clinics, emergency departments, and private specialist clinics, ${ }^{13-16}$ only one study has evaluated its use in primary care, and this study was limited to older patients (aged $\geq 65$ years) with an empirical diagnosis of pneumonia. ${ }^{17}$ No study has yet assessed the extent to which primary care clinicians routinely assess the features that make up the CRB-65 score, the value of the CRB-65 in all adults presenting with LRTI in primary care, or whether the tool can be used to predict more severe or prolonged illness in the community.

We therefore set out to explore the extent to which components of the CRB-65 rule were routinely evaluated, and to assess its validity as a tool for predicting prognosis in adults presenting with LRTI in primary care.

\section{Methods}

\section{Study subjects}

Eligible patients were aged $\geq 18$ years who presented with an illness where an acute or worsened cough was the main or dominant symptom or the clinical presentation suggested an LRTI, with a duration of $\leq 28$ days.

\section{Study design}

This was a prospective observational study in 14 primary care networks in 13 European countries with clinicians recording symptoms on presentation and management. More details on this observational Genomics to combat Resistance against Antibiotics in Community-acquired LRTI in Europe (GRACE) 01 study (www.grace-Irti.org) of acute cough have been reported elsewhere. ${ }^{18-21}$ Ethical approval for the study was obtained in each of the 13 countries in which the study was conducted.

\section{Data collection}

Clinicians recorded aspects of patients' history, symptoms, comorbidities (diabetes, chronic lung disease including chronic obstructive pulmonary disease (COPD) and cardiovascular disease), clinical findings, and their management including antibiotic prescription on a case report form (CRF). Clinicians indicated the presence or absence of 14 symptoms (cough, sputum production, shortness of breath, wheeze, coryza, fever during this illness, chest pain, muscle aching, headache, disturbed sleep, feeling generally unwell, interference with normal activities, confusion/disorientation and diarrhoea) and, if present, recorded whether each symptom constituted 'no problem', a 'mild problem', a 'moderate problem', or a 'severe problem' for the patient. In addition, clinicians had the opportunity to register the age, respiratory rate and blood pressure of patients on the CRF. In order to record routine practice, we asked clinicians only to record elements of the clinical examination (other than temperature, which clinicians were asked to record for all patients) that they would routinely check in such a patient.

Patients were given a symptom diary. They were asked to rate 13 symptoms each day until recovery (or for 28 days if symptoms were ongoing) on a 7-point scale from 'normal/not affected' to 'as bad as it can be'. Patients rated the same symptoms as the clinicians except for two symptoms - namely, confusion/disorientation and diarrhoea. They were also asked to rate the impact of their illness on their social activities, an item that was not part of the clinicians' assessment. In addition, patients were asked to indicate the day on which they felt recovered from their illness and whether or not they re-consulted with healthcare services (e.g. general practitioner [GP], nurse, or out-of-hours service) or were hospitalised for the same condition.

\section{Data analysis}

We determined the proportion of patients with CRF and diary data who had confusion, respiratory rate, blood pressure, and age recorded on the CRF. CRB-65 scores were calculated by assigning one point for each of the following: presence of confusion, respiratory rate $\geq 30 / \mathrm{min}$, systolic blood pressure $<90 \mathrm{mmHg}$ or diastolic blood pressure $\leq 60 \mathrm{mmHg}$, and age $\geq 65$ years. Patients with missing data for any of these parameters were given a missing CRB-65 score. However, in order to conduct sensitivity analyses, we also calculated CRB-65 scores 
imputing zero for all missing values.

Patients who had been hospitalised and patients who had reconsulted within the 4-week follow-up period were identified. Patients who reported one or more symptom as 'moderately bad' (scored as $\geq 3$ or more on a scale of $0-6$ ) on one or more days during the fourth week (22-28 days after the initial consultation) were identified as having a prolonged illness. We defined a CRB-65 score of $\geq 1$ as abnormal. This was based on a literature review, an assessment of the CRB-65 score frequency distribution in our dataset, and evaluation of a Receiver Operating Characteristic (ROC) curve of CRB-65 score as a predictor of prolonged illness.

As no patients died and only a small number were hospitalised, our main analysis was the association between an elevated CRB-65 score $(\geq 1)$ and prolonged illness using a twolevel hierarchical logistic regression model (with patients nested within clinicians), and controlling for antibiotic prescribing. We also examined the association between an elevated CRB-65 score and hospitalisation (using the same approach) and time to recovery, using Cox proportional hazards modelling. Sensitivity analyses were conducted by fitting the same models using the zero-imputed CRB-65 scores.

\section{Results}

A total of 3,368 participants had CRF data. The number with data on confusion, respiratory rate, blood pressure, and age and the proportion that scored positively for each of these components (i.e. had confusion, increased respiratory rate, low blood pressure, or age $\geq 65$ ) are shown in Table 1.

Diary data were available for 2,690 participants. Of these, 2,613 had symptom scores for the full 4-week follow-up period and 2,468 had data on the day of recovery. Of the 2,690 participants with diary data, a complete CRB-65 score could be calculated for only 339 (12.6\%) (complete case dataset). Imputing zero for all missing components of the CRB-65 score gave scores for all 2,690 participants (zero-imputed dataset). The proportions of participants with each CRB-65 score and participant characteristics by CRB-65 score for the complete case
Table 1. Assessment of the components of the CRB-65 prediction rule in adults with community-acquired lower respiratory tract infections presenting in primary care

\begin{tabular}{lll} 
Item & Recording, N (\%) & Positivity, N (\%) \\
\hline Confusion & $3,361(99.8)$ & $135(4.0)$ \\
\hline Respiratory rate $(\geq 30 / \mathrm{min})$ & $765(22.7)$ & $10(1.3)$ \\
\hline $\begin{array}{l}\text { Blood pressure } \\
\text { (systolic }<90 \mathrm{mmHg} \text { or a } \\
\text { diastolic } \leq 60 \mathrm{mmHg})\end{array}$ & $1,073(31.9)$ & $42(3.9)$ \\
\hline Age $(\geq 65$ years) & $3,368(100)$ & $524(15.6)$
\end{tabular}

and zero-imputed datasets are given in Tables 2 and 3, respectively. A total of 111 patients had a clinical diagnosis of pneumonia. Participants with a clinical diagnosis of pneumonia were slightly older than those with a diagnosis of non-pneumonic cough (mean 52.3 (range 49.0-55.7) years vs. 48.1 (range $47.5-48.7)$ years) and were more likely to be male (50.5\% vs. $35.4 \%$ ). Of those with a clinical diagnosis of pneumonia, a complete CRB-65 score could be calculated for 12 .

Most participants had a CRB-65 score of 0, a minority scored $1(28.0 \%$ in the complete case dataset and $21.6 \%$ in the zeroimputed dataset), and a few scored $2(2.7 \%$ in the complete case dataset and $0.7 \%$ in the zero-imputed dataset). No participants had scores of 3 or 4 . The distribution of scores between networks varied considerably in the complete case dataset. There was less inter-network variation in the distribution of CRB-65 scores in the zero-imputed dataset, although the proportion scoring 1 or 2 varied from $10 \%$ in Helsinki to $31.8 \%$ in Utrecht. The baseline symptom severity score and the proportion receiving a prescription for antibiotics increased slightly with increasing CRB-65 score.

Data on duration of symptoms were available for 2,613 participants, 314 of whom also had complete CRB-65 scores, and data on consultations during the follow-up period were available for 2,295, 312 of whom also had complete CRB-65 scores. The median duration of moderately bad symptoms in the complete case dataset was 6 days (IQR 4, 10); 20 participants

Table 2. Characteristics of 339 adults with community-acquired lower respiratory tract infections presenting in primary care with complete data for the CRB-65 score*

\begin{tabular}{|c|c|c|c|c|}
\hline & \multicolumn{4}{|c|}{ CRB-65 score } \\
\hline & 0 & 1 & 2 & All \\
\hline $\mathrm{N}($ row \%) & $235(69.3)$ & $95(28.0)$ & $9(2.7)$ & 339 \\
\hline Age, mean (SD) & $42.8(12.4)$ & $63.3(15.3)$ & $74.1(7.5)$ & $49.3(16.5)$ \\
\hline Prior duration of symptoms, median (IQR) & $4(3,7)$ & $5(3,8)$ & $4(3,6)$ & $5(3,7)$ \\
\hline Baseline symptom severity score, mean (SD) & $26.8(6.0)$ & $27.3(6.2)$ & $28.6(6.9)$ & $27.0(6.1)$ \\
\hline Antibiotics prescribed (column \%) & $165(70.2)$ & $70(73.7)$ & $7(77.8)$ & $242(71.4)$ \\
\hline Duration of moderately bad symptoms in days, median (IQR) & $6(4,9)$ & $7(4,14)$ & $7(5,14)$ & $6(4,10)$ \\
\hline Prolonged illnesst ( $\mathrm{N=334), \textrm {N } ( \% )}$ & $11(4.8)$ & $9(9.5)$ & $0(0)$ & $20(6.0)$ \\
\hline Hospitalisation $(\mathrm{N}=326), \mathrm{N}(\%)$ & $5(2.2)$ & $5(5.5)$ & $0(0)$ & $10(3.1)$ \\
\hline Day recovered, median (IQR) & $12(8,21)$ & $15(10,22)$ & $19.5(13,22)$ & $13(8,21)$ \\
\hline
\end{tabular}


Table 3. Characteristics of 2,690 adults with community-acquired lower respiratory tract infections presenting in primary care with complete or zero-imputed data for the CRB-65 score*

\begin{tabular}{|c|c|c|c|c|}
\hline & \multicolumn{4}{|l|}{ CRB-65 score } \\
\hline & 0 & 1 & 2 & All \\
\hline $\mathrm{N}(\%)$ & $2,091(77.7)$ & $580(21.6)$ & $19(0.7)$ & 2,690 \\
\hline \multicolumn{5}{|l|}{ Network, N (row \%) } \\
\hline Antwerp & $116(70.7)$ & $44(26.8)$ & $4(2.4)$ & 164 \\
\hline Balatonfüred & $275(85.9)$ & $42(13.1)$ & $3(0.9)$ & 320 \\
\hline Barcelona & $128(75.4)$ & $41(24.3)$ & $0(0)$ & 169 \\
\hline Bratislava & $268(89.6)$ & $30(10.0)$ & $1(0.3)$ & 299 \\
\hline Cardiff & $125(69.1)$ & $56(30.9)$ & $0(0)$ & 181 \\
\hline Helsinki & $81(90.0)$ & $9(10.0)$ & $0(0)$ & 90 \\
\hline Jönköping & $163(73.4)$ & $58(26.1)$ & $1(0.5)$ & 222 \\
\hline Lodz & $178(80.5)$ & $41(18.6)$ & $2(0.9)$ & 221 \\
\hline Mataró & $126(70.4)$ & $51(28.5)$ & $2(1.1)$ & 179 \\
\hline Milan & $114(74.5)$ & $38(24.8)$ & $1(0.6)$ & 153 \\
\hline Rotenberg & $144(79.6)$ & $39(19.9)$ & $1(0.55)$ & 181 \\
\hline Southampton & $125(74.4)$ & $42(25.0)$ & $1(0.6)$ & 168 \\
\hline Troms $\varnothing$ & $115(77.7)$ & $33(22.3)$ & $0(0)$ & 148 \\
\hline Utrecht & $133(68.2)$ & $59(30.3)$ & $3(1.5)$ & 195 \\
\hline Age, mean (SD) & $42.5(12.7)$ & $66.0(14.0)$ & $68.7(14.1)$ & $47.8(16.3)$ \\
\hline Prior duration of symptoms, median (IQR) & $5(3,7)$ & $6(3,10)$ & $3(2,5)$ & $5(3,8)$ \\
\hline Baseline symptom severity score, mean (SD) & $26.9(5.9)$ & $27.1(6.4)$ & $27.6(6.3)$ & $26.9(6.0)$ \\
\hline Antibiotics prescribed (column \%) & $1,124(53.8)$ & $328(56.6)$ & $12(63.2)$ & $1,464(54.4)$ \\
\hline $\begin{array}{l}\text { Duration of moderately bad symptoms in days } \\
(\mathrm{N}=2,613) \text {, median (IQR) }\end{array}$ & $6(4,11)$ & $7(4,14)$ & $7(5,14)$ & $6(4,11)$ \\
\hline Prolonged illnesst $(\mathrm{N}=2,613), \mathrm{N}(\%)$ & $170(8.4)$ & $72(12.7)$ & $1(5.6)$ & $243(9.3)$ \\
\hline Hospitalisation $(\mathrm{N}=2,545), \mathrm{N}(\%)$ & $17(0.9)$ & $11(2.1)$ & $0(0)$ & $28(1.1)$ \\
\hline Day recovered, median (IQR) & $13(8,22)$ & $15(10,29)$ & $17.5(10.5,22.5)$ & $14(9,24)$ \\
\hline
\end{tabular}

(6.0\%) had a prolonged illness (duration of moderately bad symptoms $\geq 21$ days), $173(55.5 \%)$ re-consulted, and $10(3.1 \%)$ were hospitalised. In the zero-imputed dataset the median duration of moderately bad symptoms was 6 days (IQR 4, 11); 243 (9.3\%) had a prolonged illness, 1,167 (50.9\%) re-consulted, and $28(1.1 \%)$ were hospitalised. The area under the curve of the ROC curve for the CRB-65 score predicting a prolonged illness was 0.57 (95\% Cl 0.46 to 0.68 ) using the complete case dataset and 0.54 (95\% Cl 0.51 to 0.57$)$ using the zero-imputed dataset. A cut-off point of $\geq 1$ had $45.0 \%$ sensitivity, $69.8 \%$ specificity, and positive and negative predictive values of $8.6 \%$ and $95.2 \%$, respectively, for detecting those who had a prolonged moderately severe illness in the complete case dataset and sensitivity, specificity and positive and negative predictive values of $30.0 \%, 78.5 \%, 12.5 \%$ and $91.6 \%$, respectively, in the zeroimputed dataset.

The results of regression analyses assessing the relationship between a CRB-65 score of $\geq 1$ and prolonged moderately severe illness, hospitalisation, and time to recovery, controlling for antibiotic prescribing, are given in Table 4.

An elevated CRB-65 score was not significantly associated with a prolonged moderately severe illness, controlling for antibiotic prescribing, using either a complete case analysis (OR 0.42, 95\%
$\mathrm{Cl} 0.04$ to 4.19$)$ or zero-imputed scores (OR 1.17, 95\% Cl 0.76 to 1.81) (Table 4). However, an elevated CRB-65 score was significantly associated with a reduction in the rate of recovery (hazard ratio (HR) $0.75,95 \% \mathrm{Cl} 0.64$ to 0.88 ) in the zero-imputed dataset. An elevated CRB-65 score was not significantly associated with hospitalisation (OR 2.93, 95\% Cl 0.77 to 11.17).

\section{Discussion}

\section{Summary of main findings}

Primary care clinicians recorded the components required to calculate a CRB-65 prediction score in only a minority of patients in this observational study of acute cough/LRTI in adults. Respiratory rate was recorded in less than a quarter of participants and blood pressure in less than a third. The recording of CRB-65 items varied between networks. However, the distribution of CRB-65 scores (within the zero-imputed dataset) was fairly consistent.

A CRB-65 score of $\geq 1$ was associated with a prolonged time to self-reported recovery but did not predict prolonged moderately bad symptoms ( $\geq 3$ weeks following the consultation) or hospitalisation.

\section{Strengths and limitations of the study}

The study benefited from being a purely observational study - 


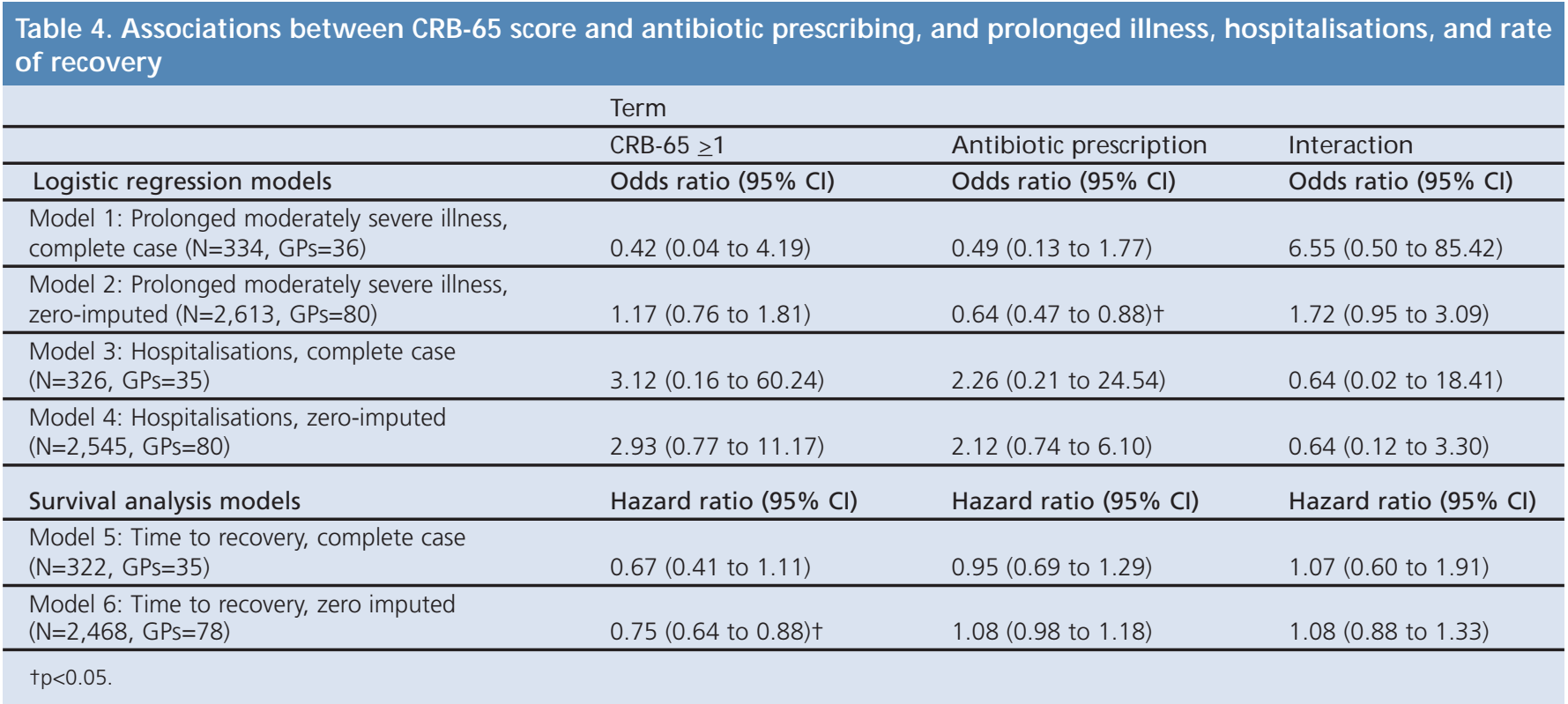

clinicians were not asked to alter their usual practice in any way and only to record items (other than age) if they would normally check them - and is therefore likely to be representative of everyday practice. It is the largest prospective observational study of LRTI to date with data from 13 European countries, and therefore should provide results that are highly generalisable. Data were collected on mortality, hospitalisations, daily symptom scores, and day of recovery, and this allowed for analyses using a number of outcomes.

Calculable CRB-65 scores were available for $12.6 \%$ of participants with follow-up data and, as such, our estimates of the distribution of CRB-65 scores - especially at the level of country-specific GP research network - do not have a high degree of precision. Furthermore, we did not have power to detect small associations between an elevated CRB-65 and poor outcomes in this dataset. For these reasons, we used zero imputation for sensitivity analyses. Given the high recording rate for confusion (99.8\%) and the universal recording of age, only respiratory rate and blood pressure scores were imputed with any frequency. The rate of positivity for these items was low (1.3\% for respiratory rate and $3.9 \%$ for blood pressure), and likely to be even lower when universally recorded (we believe clinicians are more likely to measure these parameters if the patient looks unwell, and they are more likely to be abnormal in such patients), and therefore the error rate from this approach is likely to be low. The finding that the coefficients in the analyses using the complete case and zero-imputed datasets were broadly similar supports this. Less than $5 \%$ of participants had a clinical diagnosis of pneumonia, and non-pneumonic LRTI is much less likely to be associated with systemic signs and symptoms than pneumonia. We conducted sensitivity analyses in the group with pneumonia and found no significant association. However, we did not have sufficient power to detect meaningful associations in this group. We also conducted a sensitivity analysis using re-consultation within 4 weeks as an outcome and again found no significant association between elevated CRB-65 scores and outcome.

\section{Comparison with existing literature}

No other study has examined the value of CRB-65 in predicting prolonged illness in primary care. One primary care-based study assessing the discriminatory value of CRB-65 for predicting mortality in older patients ( $\geq 65$ years) with CAP found a 30-day mortality rate of $3.5 \%$ and good evidence for the discriminatory value of CRB-65. ${ }^{17}$

Elevated CRB-65 scores $(\geq 1)$ resulted primarily from age $\geq 65$ years (positivity for confusion, elevated respiratory rate and low blood pressure were low in this study). Age has been found to be a predictor of death and hospitalisation in other studies. ${ }^{22-24}$ However, it is still not clear whether age can be used as an independent predictor of prolonged time to recovery or whether the association between CRB-65 (driven largely by age) and prolonged time to recovery observed in this study (using zero imputation) is confounded by other risk factors such as comorbid conditions.

An alternative prognostic rule for older patients (aged $\geq 65$ years) with LRTI in the community based on an assessment of seven easy-to-measure characteristics (diagnosis, age, congestive heart failure, diabetes, using oral glucocorticoids, hospitalisations in previous year, and use of antibiotics in previous month) has been found to have reasonable performance (area under the ROC curve $=0.75$ ) in predicting death or hospitalisation within 30 days of diagnosis. ${ }^{25}$ The same rule has been shown to have similar properties in older diabetic patients ${ }^{26}$ but has not yet been evaluated in younger patients.

\section{Implications for future research and} clinical practice

We found that respiratory rate and blood pressure are measured infrequently in patients presenting in primary care with LRTI. 
Routine CRB-65 assessment would therefore require a change in the routine clinical assessment of most patients with acute LRTI. However, it is not yet clear whether GPs are good at identifying patients in whom these parameters should be measured or, if they were measured universally, whether they would be more predictive. We found no good evidence that the CRB-65 score is valuable in the assessment of patients with LRTI in the community. However, an elevated CRB-65 score mediated largely through scoring a point for age $\geq 65$ years was associated with a prolonged time to recovery when using zero imputation. Further prognostic studies are needed to assess the value of age in multivariable analyses that include a wider range of potential explanatory variables and that aim to produce a new rule for predicting prolonged illness for LRTI in primary care. Larger studies will be needed to assess the value of CRB-65 in predicting mortality and hospitalisations, especially in patients where a GP suspects pneumonia.

\section{Handling editor Hilary Pinnock Statistical review Gopal Netuveli}

Acknowledgements The authors acknowledge the essential contribution of the entire GRACE team including partners, management, and primary care networks, whose imagination and hard work have made this study possible. They also acknowledge the essential contribution of participants in the GRACE-01 cohort study.

Conflicts of interest The authors declare that they have no conflicts of interest in relation to this article.

Contributorship The GRACE project was conceived and developed by HG, $\mathrm{CB}, \mathrm{SC}, \mathrm{KH}, \mathrm{PL}$, and TV. CB was the chief investigator of the GRACE-01 study. This analysis was conceived by SC and NF. NF conducted the analyses, with help from $\mathrm{SC}, \mathrm{KH}, \mathrm{JC}$, and $\mathrm{CB}$. All authors contributed to the writing of the manuscript and reviewed the final draft.

Funding This work was supported by the 6th Framework Programme of the European Commission (reference: LSHM-CT-2005-518226). The South East Wales Trials Unit is funded by the National Institute for Social Care and Health Research in Wales, and in Flanders (Belgium) this work was supported by the Research Foundation, Flanders (G.F0274.08N).

\section{References}

1. Metlay JP, Fine MJ. Testing strategies in the initial management of patients with community-acquired pneumonia. Ann Intern Med 2003;138:109-18.

2. Butler CC, Rollnick S, Kinnersley P, Jones A, Stott N. Reducing antibiotics for respiratory tract symptoms in primary care: consolidating 'why' and considering 'how'. Br J Gen Pract 1998;48:1865-70.

3. Costelloe C, Metcalfe C, Lovering A, Mant D, Hay AD. Effect of antibiotic prescribing in primary care on antimicrobial resistance in individual patients: systematic review and meta-analysis. BMJ 2010;340:c2096. http://dx.doi.org/10.1136/bmj.c2096

4. Malhotra-Kumar S, Lammens C, Coenen S, Van Herck K, Goossens H. Effect of azithromycin and clarithromycin therapy on pharyngeal carriage of macrolideresistant streptococci in healthy volunteers: a randomised, double-blind, placebocontrolled study. Lancet 2007;369:482-90.

http://dx.doi.org/10.1016/S0140-6736(07)60235-9

5. Pinnock $H$, Thomas $M$, Tsiligianni l, et al. The International Primary Care Respiratory Group (IPCRG) Research Needs Statement 2010. Prim Care Respir J 2010;19(Suppl 1):S1-S20. http://dx.doi.org/10.4104/pcrj.2010.00021

6. Lim WS, Lewis S, Macfarlane JT. Severity prediction rules in community acquired pneumonia: a validation study. Thorax 2000;55:219-23. http://dx.doi.org/10.1136/thorax.55.3.219

7. Lim WS, van der Eerden MM, Laing $R$, et al. Defining community acquired pneumonia severity on presentation to hospital: an international derivation and validation study. Thorax 2003;58:377-82. http://dx.doi.org/10.1136/ thorax.58.5.377

8. Lim WS, Baudouin SV, George RC, et al. BTS guidelines for the management of community acquired pneumonia in adults: update 2009. Thorax 2009;64(Suppl 3):iii1-55. http://dx.doi.org/10.1136/thx.2009.121434

9. Ewig S, Torres A. Severity scores for CAP. Much workload for the next bias. Thorax 2010;65:853-5. http://dx.doi.org/10.1136/thx.2010.143297

10. Chalmers JD, Singanayagam A, Akram AR, et al. Severity assessment tools for predicting mortality in hospitalised patients with community-acquired pneumonia. Systematic review and meta-analysis. Thorax 2010;65:878-83. http://dx.doi.org/10.1136/thx.2009.133280

11. Loke YK, Kwok CS, Niruban A, Myint PK. Value of severity scales in predicting mortality from community-acquired pneumonia: systematic review and metaanalysis. Thorax 2010;65:884-90. http://dx.doi.org/10.1136/thx.2009.134072

12. McNally M, Curtain J, O'Brien KK, Dimitrov BD, Fahey T. Validity of British Thoracic Society guidance (the CRB-65 rule) for predicting the severity of pneumonia in general practice: systematic review and meta-analysis. $\mathrm{Br} J$ Gen Pract 2010;60:e423-33. http://dx.doi.org/10.3399/bjgp10X532422

13. Bauer $\Pi$, Ewig $S$, Marre R, Suttorp N, Welte T. CRB-65 predicts death from community-acquired pneumonia. J Intern Med 2006;260:93-101. http://dx.doi.org/10.1111/j.1365-2796.2006.01657.x

14. Capelastegui A, Espana PP, Quintana JM, et al. Validation of a predictive rule for the management of community-acquired pneumonia. Eur Respir J 2006;27:151-7. http://dx.doi.org/10.1183/09031936.06.00062505

15. Kruger S, Ewig S, Marre R, et al. Procalcitonin predicts patients at low risk of death from community-acquired pneumonia across all CRB-65 classes. Eur Respir J 2008;31:349-55. http://dx.doi.org/10.1183/09031936.00054507

16. Man SY, Lee $N$, Ip M, et al. Prospective comparison of three predictive rules for assessing severity of community-acquired pneumonia in Hong Kong. Thorax 2007;62:348-53. http://dx.doi.org/10.1136/thx.2006.069740

17. Bont J, Hak E, Hoes AW, Macfarlane JT, Verheij TJ. Predicting death in elderly patients with community-acquired pneumonia: a prospective validation study reevaluating the CRB-65 severity assessment tool. Arch Intern Med 2008;168:1465-8. http://dx.doi.org/10.1001/archinte.168.13.1465

18. Butler CC, Hood K, Verheij T, et al. Variation in antibiotic prescribing and its impact on recovery in patients with acute cough in primary care: prospective study in 13 countries. BMJ 2009;338:b2242. http://dx.doi.org/10.1136/ bmj.b2242

19. Stanton N, Hood K, Kelly MJ, et al. Are smokers with acute cough in primary care prescribed antibiotics more often, and to what benefit? An observational study in 13 European countries. Eur Respir J 2010;35:761-7. http://dx.doi.org/10.1183/09031936.00168409

20. Jakobsen KA, Melbye H, Kelly MJ, et al. Influence of CRP testing and clinical findings on antibiotic prescribing in adults presenting with acute cough in primary care. Scand J Prim Health Care 2010;28:229-36. http://dx.doi.org/10.3109/02813432.2010.506995

21. Butler CC, Hood K, Kelly MJ, et al. Treatment of acute cough/lower respiratory tract infection by antibiotic class and associated outcomes: a 13 European country observational study in primary care. J Antimicrob Chemother 2010;65:2472-8. http://dx.doi.org/10.1093/jac/dkq336

22. Fine MJ, Auble TE, Yealy DM, et al. A prediction rule to identify low-risk patients with community-acquired pneumonia. N Engl J Med 1997;336:243-50. http://dx.doi.org/10.1056/NEJM199701233360402

23. Mortensen EM, Coley CM, Singer DE, et al. Causes of death for patients with community-acquired pneumonia: results from the Pneumonia Patient Outcomes Research Team cohort study. Arch Intern Med 2002;162:1059-64. http://dx.doi.org/10.1001/archinte.162.9.1059

24. Hak E, Wei F, Nordin J, Mullooly J, Poblete S, Nichol KL. Development and validation of a clinical prediction rule for hospitalization due to pneumonia or influenza or death during influenza epidemics among community-dwelling elderly persons. J Infect Dis 2004;189:450-8. http://dx.doi.org/10.1086/381165

25. Bont J, Hak E, Hoes AW, Schipper M, Schellevis FG, Verheij TJ. A prediction rule for elderly primary-care patients with lower respiratory tract infections. Eur Respir J 2007;29:969-75. http://dx.doi.org/10.1183/09031936.00129706

26. Venmans LM, Bont J, Gorter KJ, Verheij TJ, Rutten GE, Hak E. Prediction of complicated lower respiratory tract infections in older patients with diabetes. $\mathrm{Br} J$ Gen Pract 2008;58:564-8. http://dx.doi.org/10.3399/bjgp08X319620 